\title{
Women's Preferences for Sexual Dimorphism in Faces: Data from a Sample of Arab Women
}

\author{
Sarah A. H. Alharbi ${ }^{1} \cdot$ Iris J. Holzleitner ${ }^{1} \cdot$ Anthony J. Lee $^{2} \cdot$ S. Adil Saribay ${ }^{3} \cdot$ Benedict C. Jones $^{1}$
}

Received: 31 March 2020 / Revised: 1 May 2020 / Accepted: 5 May 2020 / Published online: 14 May 2020

(C) The Author(s) 2020

\begin{abstract}
Many previous studies have investigated the effects of manipulating sexually dimorphic shape characteristics in face images on attractiveness judgments. However, results have been mixed and show considerable cross-cultural variability, particularly for women's judgments of men's facial attractiveness. Because very little research has investigated face preferences in Arab samples, we assessed Arab women's preferences for sexually dimorphic face shapes (study 1) and the effect of sexually dimorphic face shapes on Arab women's dominance perceptions (study 2). Analyses showed that Arab women preferred feminized versions of both women's and men's faces over masculinized versions (study $1, N=272$ ) and that masculinizing face shape had a positive effect on Arab women's perceptions of the dominance of men, but not women (study 2, $N=270$ ). These image manipulations did not have a significant effect on perceptions of trustworthiness, however (study $3, N=434$ ). Collectively, these results suggest that Arab women prefer relatively feminine face shapes in potential mates that they perceive as being low dominance. We discuss some directions for future research on the ultimate function of Arab women's preferences for sexual dimorphism in faces
\end{abstract}

Keywords Faces $\cdot$ Attractiveness $\cdot$ Mate preferences $\cdot$ Culture

\section{Introduction}

Many researchers have suggested that exaggerated sex-typical characteristics in faces (masculine characteristics in men's faces and feminine characteristics in women's faces) advertise good health (Thornhill and Gangestad 1999; Little et al. 2011). However, empirical tests of this hypothesis have produced mixed results, with some studies reporting significant correlations between exaggerated sex-typical characteristics and health measures (e.g., Thornhill and Gangestad 2006), while others either did not (e.g., Cai et al. 2019; Foo et al. 2017) or reported significant correlations for only one sex

Sarah A. H. Alharbi

s.alharbi.1@research.gla.ac.uk

1 Institute of Neuroscience \& Psychology, University of Glasgow, Scotland, UK

2 Division of Psychology, University of Stirling, Scotland, UK

3 Department of Psychology, Boğaziçi Üniversitesi, Istanbul, Turkey (e.g., Rhodes et al. 2003). By contrast with these mixed results for health and sexually dimorphic facial characteristics, studies have consistently found that feminized versions of images of men's and women's faces are ascribed pro-social traits, such as emotional warmth and trustworthiness, while masculinized versions are ascribed anti-social traits, such as dominance and aggressiveness (Boothroyd et al. 2007; Perrett et al. 1998).

Because both health and personality are important for attraction (Little et al. 2011), many studies have investigated how feminizing versus masculinizing shape characteristics in face images influences attractiveness judgments. While these studies have consistently reported positive effects of feminizing shape characteristics on attractiveness judgments of women's faces (Jones et al. 2018; Little et al. 2011; Perrett et al. 1998), findings are considerably more mixed for judgments of men's facial attractiveness (Little et al. 2011). Moreover, evidence suggests that women's judgments of men's facial attractiveness may vary considerably across cultures. For example, studies of men's facial attractiveness conducted in the UK, Japan, and Bangladesh have typically re- 
ported that women prefer versions of men's faces with relatively feminine shapes (e.g., De Barra et al. 2013; PentonVoak et al. 1999; Perrett et al. 1998; but see Jones et al. 2018). By contrast, studies of men's facial attractiveness conducted in the USA and Jamaica have typically reported that women prefer versions of men's faces with relatively masculine shapes (e.g., Johnston et al. 2001; Penton-Voak et al. 2004; Rennels et al. 2008).

Several previous studies have attempted to link cultural differences in women's preferences for male faces with masculinized versus feminized shape characteristics to regional differences in ecological conditions. For example, some studies have reported that women show stronger preferences for masculinity in men's faces in countries where health is poor (DeBruine et al. 2010), violent crime commonplace and income inequality pronounced (Brooks et al. 2011), or where environmental conditions were favorable, as opposed to harsh (Marcinkowska et al. 2019). Other work has found that women in more industrialized societies show stronger preferences for masculine men (Scott et al. 2014). However, these findings for ecological conditions and masculinity preferences have not replicated well across studies (Brooks et al. 2011; DeBruine et al. 2010; Marcinkowska et al. 2019; Scott et al. 2014).

To date, Arab women's preferences for sexually dimorphic shape characteristics in face images have not been well documented. Of the cross-cultural studies of women's facial masculinity preferences (i.e., those testing women from many geographic regions), only Marcinkowska et al. (2019) included a small sample of Arab women (21 Saudi Arabian women). This sample showed a preference for feminized versions of male faces over masculinized versions. This relative lack of comprehensive data on Arab women's preferences may reflect the well-established tendency for studies of face preferences to focus mostly on preferences in Western Europe and North America (Scott et al. 2014). Consequently, we investigated 272 Arab women's preferences for masculinized versus feminized versions of images of male and female faces (study 1).

Research on perceptions of masculinized versus feminized versions of face shapes have found that masculinized faces are perceived as more dominant than feminized versions, particularly when assessing men's dominance (e.g., Perrett et al. 1998). To establish whether Arab women preferred faces of men judged to be high or low dominance, study 2 investigated whether masculinizing face shapes increased Arab women's perceptions of dominance. We know of no previous research that has investigated the effects of sexually dimorphic face shapes on dominance perceptions in an Arab sample.

Finally, in study 3 , we investigated perceptions of the trustworthiness of masculinized versus feminized versions of face shapes. Previous research on Western and Japanese samples has found that feminized faces are typically judged to be more trustworthy than masculinized faces (Perrett et al. 1998). Here, we investigated whether this pattern also holds for a sample of Arab women.

\section{Study 1: Attractiveness}

\section{Methods}

\section{Participants}

Two hundred and seventy-two Arab women (mean age = 31.68 years, $\mathrm{SD}=8.15$ years) took part in this online study. Participants were recruited by following links to an online study of facial attractiveness posted on Saudi Arabian social media and were recruited from Saudi Arabia, Jordan, Oman, Egypt, United Arab Emirates, and Kuwait. The study was administered via faceresearch.org (DeBruine 2019).

\section{Stimuli}

Stimuli were manufactured using the same methods used in previous studies of preferences for sexual dimorphism in face shape (e.g., DeBruine et al. 2010; Jones et al. 2018; Perrett et al. 1998), from an open access set of Turkish face images (Saribay et al. 2018), and using standard computer graphic methods implemented in webmorph.org (DeBruine 2018; Tiddeman et al. 2001). Eleven images of people wearing glasses or headscarves that obscured the face were removed from the image set.

First, we manufactured a female face prototype by averaging the shape, color, and texture information from 142 female face images. Next, we manufactured a male face prototype by averaging the shape, color, and texture information from 111 male face images. Finally, we created masculinized and feminized versions of 60 of the individual face images (30 male, 30 female) by adding or subtracting $50 \%$ of the differences in 2D shape between the male and female prototypes to each of 60 individual faces randomly selected from the full image set. Examples of these manipulations applied to the male and female prototypes are shown in Fig. 1.

This process created 60 pairs of faces ( 30 male and 30 female pairs), with each pair consisting of a masculinized and feminized version of a given face.

\section{Procedure}

The sixty pairs of faces were shown in a fully randomized order, with the side of the screen on which the masculinized and feminized versions were presented also fully randomized. Participants were instructed to click on the face in each pair 
Fig. 1 Examples of the sexual dimorphism transform applied to male (top row) and female (bottom row) face prototypes. Masculinized versions are shown in the left column and feminized versions in the right column. We show example of the shape transforms applied to prototype faces because we do not have permission to show individual face images
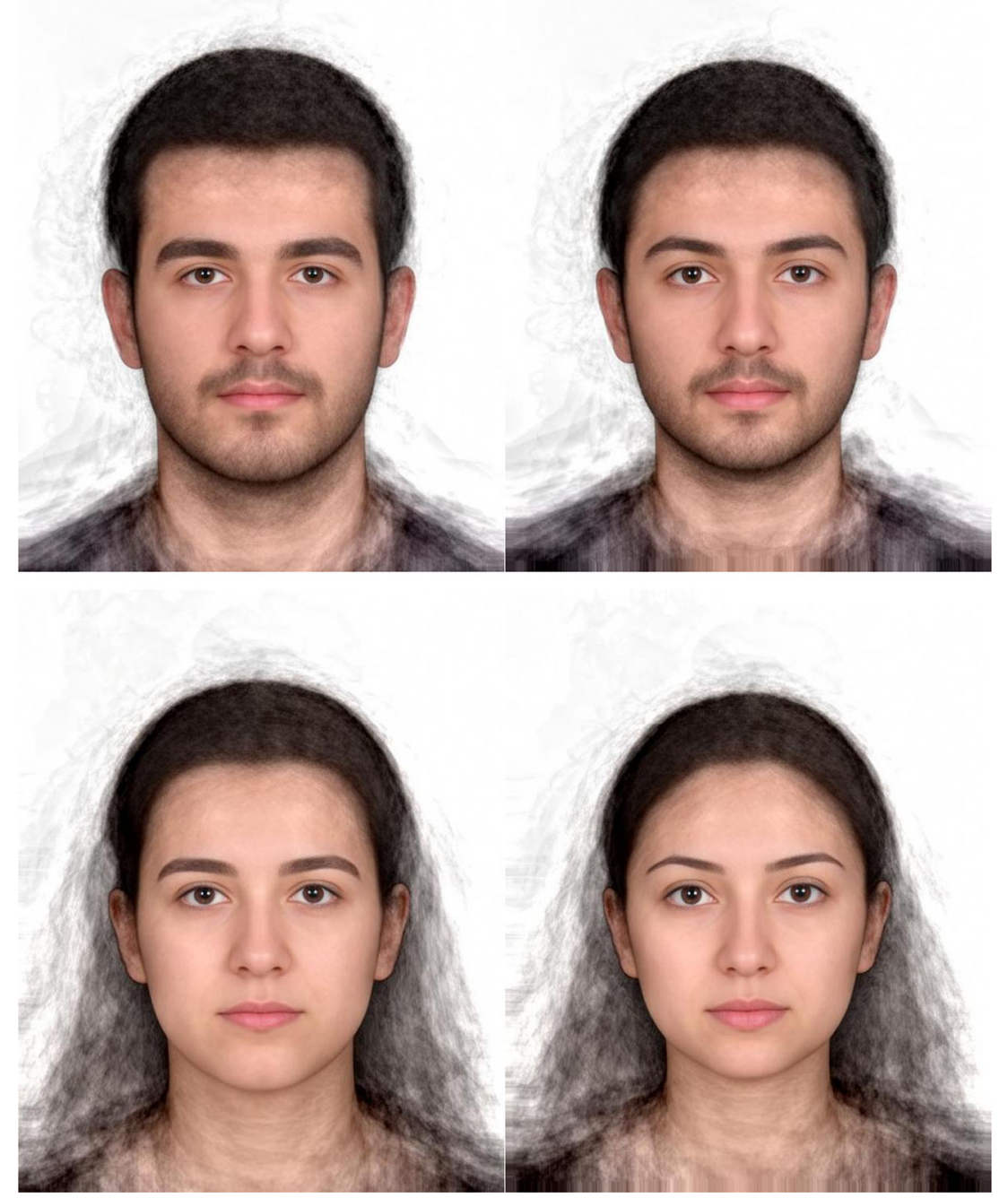

they thought was more attractive. Instructions were presented in Arabic. A back-translated version of the instructions confirmed that our initial translation of instructions from English to Arabic was accurate.

Some recent research suggests that forced-choice paradigms can produce qualitatively different patterns of results than other methods for assessing preferences for sexually dimorphic face-shape characteristics (Jones and Jaeger 2019). However, we used the forced choice method in the current study to allow our results to be directly compared with the previous research discussed in our "Introduction".

\section{Results}

All data, output, and analysis code are publicly available on the Open Science Framework (osf.io/24tjz/). Preferences were analyzed with binomial mixed effects models using the lme4 (Bates et al. 2015) and lmerTest (Kuznetsova et al. 2015) package in the R statistical software (R Core Team 2013).
Participant's choice was the dependent variable, coded such that greater scores indicated a greater preference for femininity ( chose masculinized face $=0$, chose feminized face $=1$ ). Participant age ( $z$-standardized at the participant level) and sex of face (effect code: male $=-0.5$, female $=0.5$ ) were added as predictors. Random slopes were specified maximally (Barr et al. 2013).

In this initial analysis, the intercept was significant and positive (estimate $=0.81, \mathrm{SE}=0.11, z=7.64, p<0.001$ ). Converting this estimate to proportions, this equates to women choosing, on average, the feminized version of faces as the more attractive face on $69 \%$ of trials. Neither the main effect of participant age (estimate $=0.05, \mathrm{SE}=0.06, z=0.75, p=$ 0.45 ) nor the main effect of sex of face (estimate $=0.23, \mathrm{SE}=$ $0.18, z=1.26, p=0.21$ ) was significant.

When male and female faces were analyzed separately, the intercepts were both significant (female faces: estimate $=0.97$, $\mathrm{SE}=0.15, z=6.63, p<0.001 ;$ male faces: estimate $=0.65, \mathrm{SE}$ $=0.13, z=4.87, p<0.001)$. This equates to, on average, women choosing the feminized version of female faces as 
the more attractive face on $73 \%$ of trials and choosing the feminized version of male faces as the more attractive face on $66 \%$ of trials. The effect of participant age was not significant for either sex of face (female faces: estimate $=-0.01, \mathrm{SE}$ $=0.07, z=-0.16, p=0.88$; male faces: estimate $=0.14, \mathrm{SE}=$ $0.08, z=1.87, p=0.06$ ). These results are summarized in Fig. 2.

\section{Study 2: Dominance}

\section{Methods}

Stimuli, recruitment, and testing procedure were identical to those used in study 1 , except that we asked participants to click on the person who looked more dominant and tested 270 women (mean age $=25.72$ years, $\mathrm{SD}=6.39$ years).

\section{Results}

Analyses were identical to those used in study 1. In our initial analysis of dominance perceptions, the intercept was significant and negative (estimate $=-0.20, \mathrm{SE}=0.09, z=-2.37, p<$ $0.05)$. Converting this estimate to proportions, this equates to women choosing, on average, the feminized version of faces as the more dominant face on $45 \%$ of trials. The main effect of sex of face was significant (estimate $=0.55, \mathrm{SE}=0.08, z=$ $6.90, p<0.001$ ), indicating that the effect of sexually dimorphic face shapes on dominance perceptions was greater for male than female faces. The main effect of participant age was not significant (estimate $=-0.04, \mathrm{SE}=0.08, z=-$ $0.45, p=0.65$ ).

When male and female faces were analyzed separately, the intercept was significant for male (estimate $=-0.47, \mathrm{SE}=$ $0.09, z=-5.02, p<0.001)$, but not female faces. This equates to, on average, women choosing the feminized version of male faces as the more dominant on $38 \%$ of trials and choosing the feminized version of female faces as the more dominant on $52 \%$ of trials. The effect of participant age was not significant for either sex of face (female faces: estimate $=-$ $0.01, \mathrm{SE}=0.09, z=-0.08, p=0.94$; male faces: estimate $=-$ $0.05, \mathrm{SE}=0.08, z=-0.7, p=0.51)$. These results are summarized in Fig. 3.

\section{Study 3: Trustworthiness}

\section{Methods}

Stimuli, recruitment, and testing procedure were identical to those used in study 1 and study 2, except that we asked participants to click on the person who looked more trustworthy and tested 434 women (mean age $=23.88$ years, $\mathrm{SD}=6.11$ years).

\section{Results}

Analyses were identical to those used in study 1 and study 2 . The analysis revealed no significant effects (all absolute estimates $<0.15$, all SE $<0.13$, all absolute $z<1.15$, all $p>0.25$ ). These results indicate that our participants' perceptions of
Fig. 2 Violin plots showing distributions of the proportion of feminized versions of male and female faces chosen as more attractive by Arab women. Dots and lines show mean and 95\% confidence intervals respectively

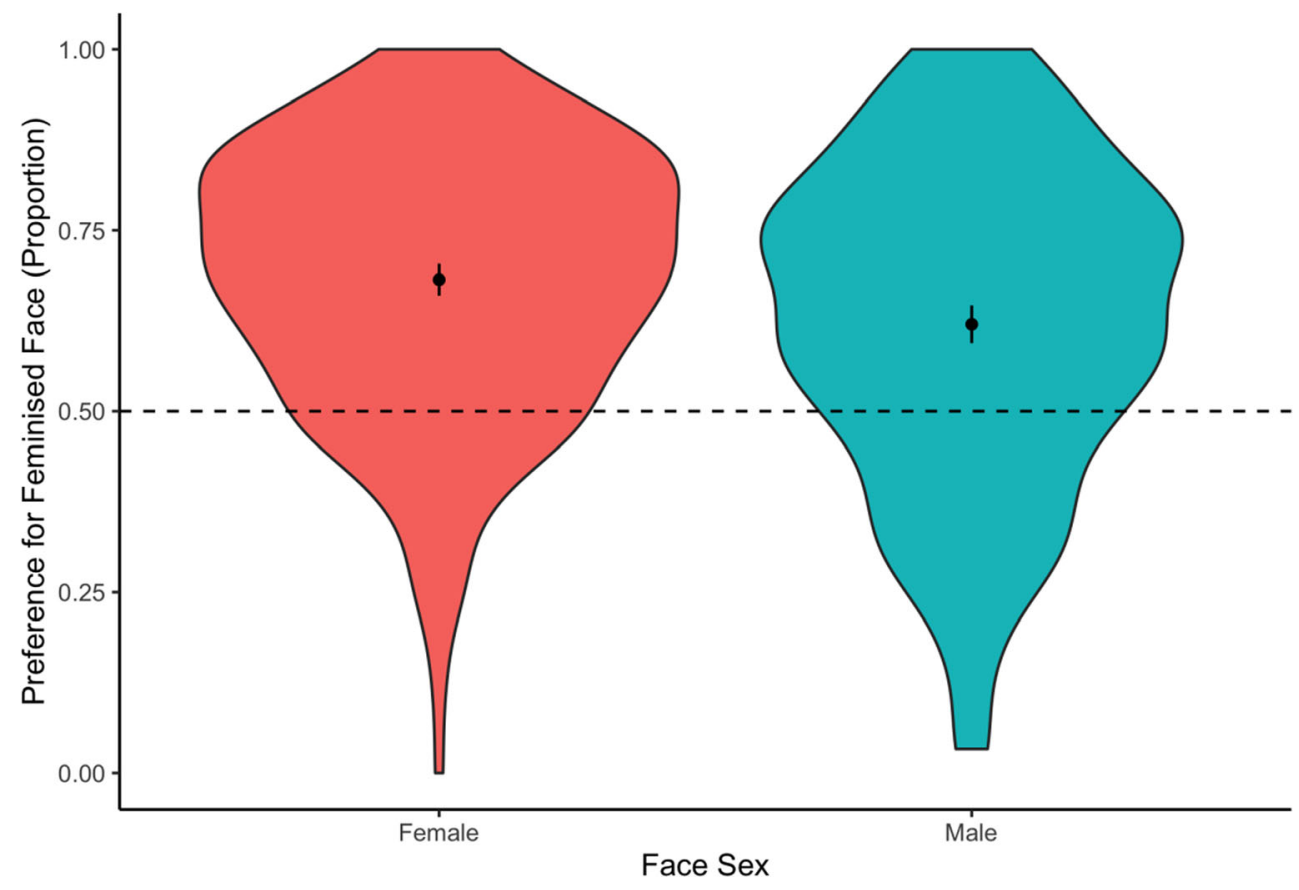


Fig. 3 Violin plots showing distributions of the proportion of feminized versions of male and female faces chosen as more dominant by Arab women. Dots and lines show mean and 95\% confidence intervals respectively

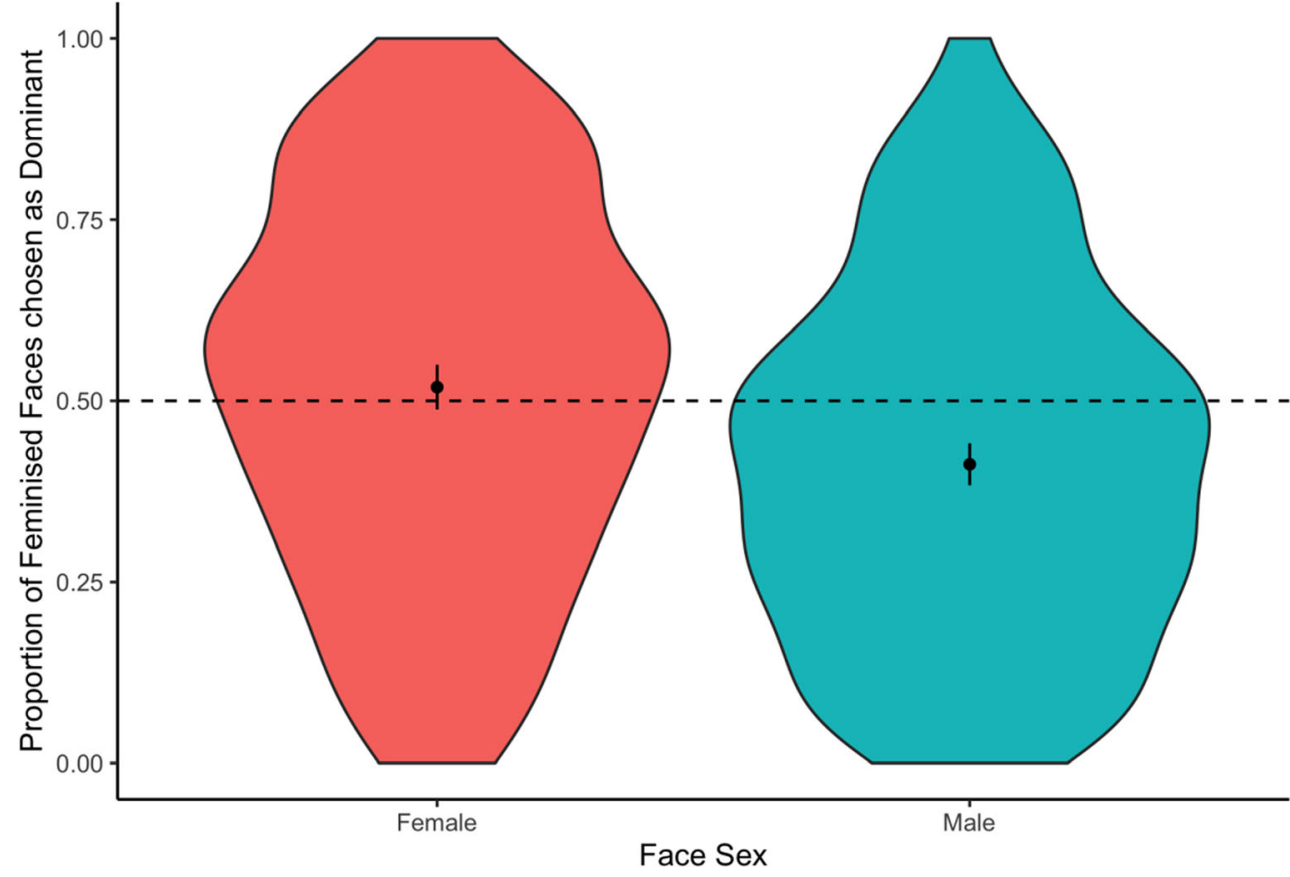

images. In this study, Arab women preferred feminized over masculinized versions for judgments of both male and female faces. These results are similar to women's preferences for facial femininity in studies of UK, Bangladeshi, and Japanese women's preferences, which also showed clear femininity preferences (e.g., De Barra et al. 2013; Penton-Voak et al. 1999; Perrett et al. 1998; but see Jones et al. 2018). They are also consistent with the results of a previous study of 21 Arab women's preferences (Marcinkowska et al. 2019), which
Fig. 4 Violin plots showing distributions of the proportion of feminized versions of male and female faces chosen as more trustworthy by Arab women. Dots and lines show mean and 95\% confidence intervals respectively

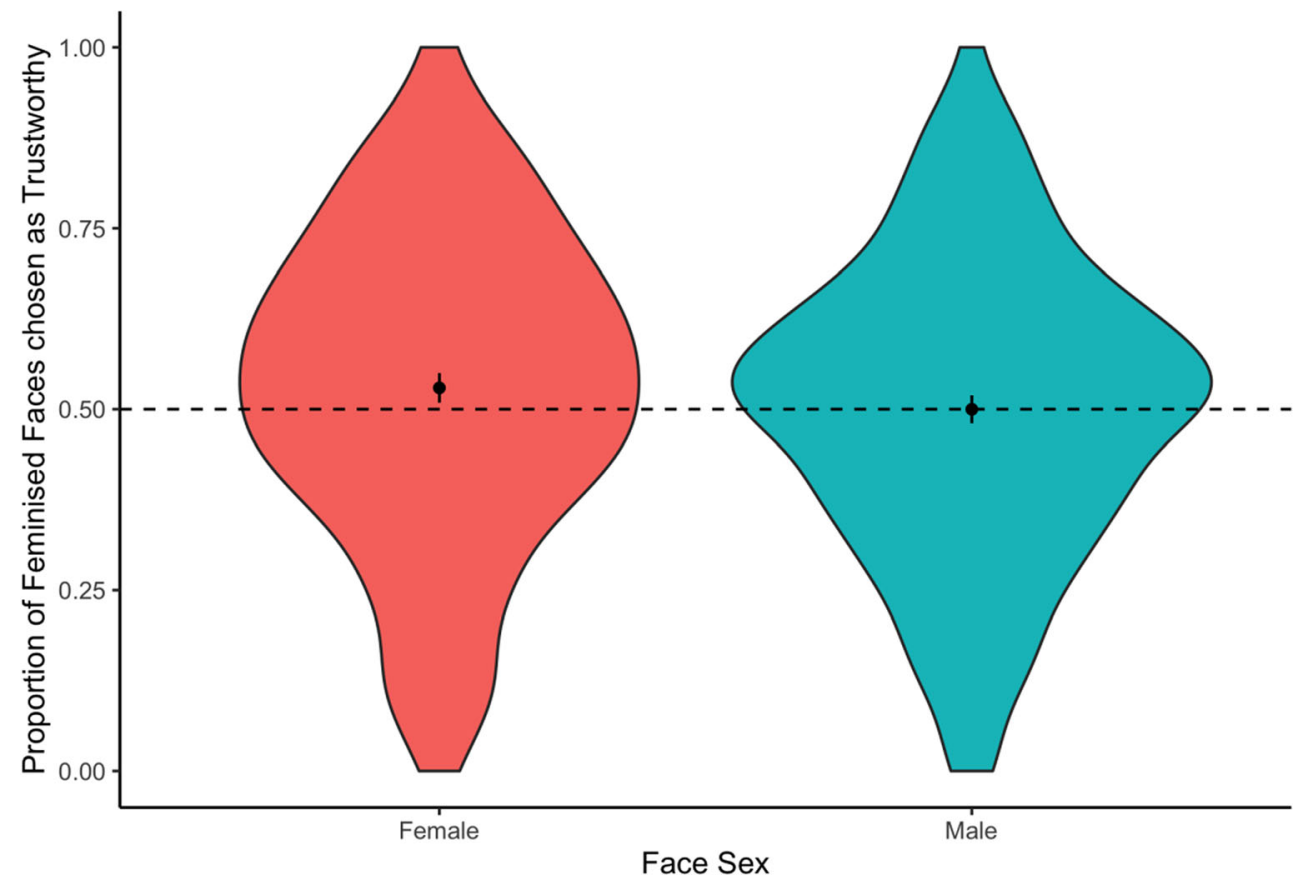


found that Arab women preferred feminized versions of male face images over masculinized versions. Collectively, these results suggest that UK, Bangladeshi, Japanese, and Arab women prefer male faces displaying shape characteristics associated with feminine shape characteristics.

As mentioned above, our results are consistent with some previous studies of women's judgments of men's facial attractiveness (e.g., De Barra et al. 2013; Marcinkowska et al. 2019; Penton-Voak et al. 1999; Perrett et al. 1998; but see Jones et al. 2018). However, they differ from those of studies of US and Jamaican women's face preferences, which reported that women tended to prefer masculinized versions of men's faces over feminized versions (e.g., Johnston et al. 2001; PentonVoak et al. 2004; Rennels et al. 2008). Although some work has recently demonstrated that women's face preferences can differ according to the type of paradigm used to assess preferences (Jones and Jaeger 2019), this effect of testing paradigm is unlikely to explain this difference in preferences across studies: all of the studies described above employed similar forced-choice paradigms.

In study 2 , we found that masculinizing male, but not female, faces increased Arab women's $(N=270)$ perceptions of others' dominance. These results are consistent with previous research reporting that masculinity had stronger positive effects on dominance perceptions of male than female faces (e.g., Perrett et al. 1998). Together with our results for face preferences in study 1, these results suggest that Arab women tend to find the faces of potential mates that they consider low dominance particularly attractive.

Study 3 found no evidence that masculinizing shape characteristics influenced Arab women's $(N=434)$ perceptions of trustworthiness. This null result is noteworthy for two reasons. First, most previous studies have found that feminizing face images increased perceived trustworthiness (e.g., Perrett et al. 1998). While these results have been reported for Western and Japanese samples, study 3's null results suggest that this effect of femininity on trustworthiness is not a universal characteristic of social perception. Second, study 3's null results suggest that Arab women's preferences for feminized, rather than masculinized, male faces is not due to feminine men being perceived to be relatively trustworthy.

Why might Arab women show strong preferences for feminized versions of men's faces? One possibility is that this pattern is a consequence of Arab women showing relatively low interest in uncommitted sexual relationships in combination with the tendency for women with low interest in casual sex to prefer more feminine men as potential mates. Indeed, Marcinkowska et al. (2019) have previously found that Arab women do score relatively low on the Sociosexual Orientation Inventory (a widely used measure of openness to uncommitted sexual relationships, Penke and Asendorpf 2008 ) and that women in geographic regions where interest in uncommitted sexual relationships is low tend to show stronger preferences for feminine men. Although plausible, more work is needed to explore this potential explanation.

Acknowledgments The authors thank Ziad Choueiki for helping in translating participant instructions.

Data Availability All data and analysis code are publicly available at https://osf.io/24tjz/.

Open Access This article is licensed under a Creative Commons Attribution 4.0 International License, which permits use, sharing, adaptation, distribution and reproduction in any medium or format, as long as you give appropriate credit to the original author(s) and the source, provide a link to the Creative Commons licence, and indicate if changes were made. The images or other third party material in this article are included in the article's Creative Commons licence, unless indicated otherwise in a credit line to the material. If material is not included in the article's Creative Commons licence and your intended use is not permitted by statutory regulation or exceeds the permitted use, you will need to obtain permission directly from the copyright holder. To view a copy of this licence, visit http://creativecommons.org/licenses/by/4.0/.

\section{References}

Barr, D. J., Levy, R., Scheepers, C., \& Tily, H. J. (2013). Random effects structure for confirmatory hypothesis testing: Keep it maximal. Journal of Memory and Language, 68(3), 255-278. https://doi. org/10.1016/j.jml.2012.11.001.

Bates, D., Mächler, M., Bolker, B. M., \& Walker, S. C. (2015). Fitting linear mixed-effects models usng lme4. Journal of Statistical Software, 67(1), 1-48.

Boothroyd, L. G., Jones, B. C., Burt, D. M., \& Perrett, D. I. (2007). Partner characteristics associated with masculinity, health and maturity in male faces. Personality and Individual Differences, 43(5), $1161-1173$.

Brooks, R., Scott, I. M., Maklakov, A. A., Kasumovic, M. M., Clark, A. P., \& Penton-Voak, I. S. (2011). National income inequality predicts women's preferences for masculinized faces better than health does. Proceedings of the Royal Society B, 278(1707), 810-812.

Cai, Z., Hahn, A. C., Zhang, W., Holzleitner, I. J., Lee, A. J., DeBruine, L. M., \& Jones, B. C. (2019). No evidence that facial attractiveness, femininity, averageness, or coloration are cues to susceptibility to infectious illnesses in a university sample of young adult women. Evolution and Human Behavior, 40(2), 156-159.

Core Team, R. (2013). A language and environmental for statistical computing. Vienna: R Foundation for Statistical Computing.

De Barra, M., DeBruine, L. M., Jones, B. C., Mahmud, Z. H., \& Curtis, V. A. (2013). Illness in childhood predicts face preferences in adulthood. Evolution and Human Behavior, 34(6), 384-389.

DeBruine, L. M. (2018). debruine/webmorph: Beta release 2 (Version v0.0.0.9001). Zenodo. https://doi.org/10.5281/zenodo.1162670

DeBruine, L. M. (2019). debruine/experimentum: Beta release 1 (Version v.0.1). Zenodo. https://doi.org/10.5281/zenodo.2634356

DeBruine, L. M., Jones, B. C., Crawford, J. R., Welling, L. L., \& Little, A. C. (2010). The health of a nation predicts their mate preferences: cross-cultural variation in women's preferences for masculinized male faces. Proceedings of the Royal Society B: Biological Sciences, 277, 2405-2410. https://doi.org/10.1098/rspb.2009.2184

Foo, Y. Z., Simmons, L. W., \& Rhodes, G. (2017). Predictors of facial attractiveness and health in humans. Scientific Reports, 7, 39731.

Johnston, V. S., Hagel, R., Franklin, M., Fink, B., \& Grammer, K. (2001). Male facial attractiveness: evidence for hormone-mediated adaptive design. Evolution and Human Behavior, 22(4), 251-267. 
Jones, A. L., \& Jaeger, B. (2019). Biological bases of beauty revisited: the effect of symmetry, averageness, and sexual dimorphism on female facial attractiveness. Symmetry, 11(2), 279.

Jones, B. C., Hahn, A. C., Fisher, C. I., Wang, H., Kandrik, M., Han, C., et al. (2018). No compelling evidence that preferences for facial masculinity track changes in women's hormonal status. Psychological Science, 29, 996-1005. https://doi.org/10.1177/ 0956797618760197.

Kuznetsova, A., Brockhoff, P. B., \& Christensen, R. H. B. (2015). lmerTest: tests for random and fixed effects for linear mexed effect models. Retrieved from https://CRAN.R-project.org/package $=$ lmerTest

Little, A. C., Jones, B. C., \& DeBruine, L. M. (2011). Facial attractiveness: evolutionary based research. Philosophical Transactions of the Royal Society, B: Biological Sciences, 366(1571), 1638-1659.

Marcinkowska, U. M., Rantala, M. J., Lee, A. J., Kozlov, M. V., Aavik, T., Cai, H., et al. (2019). Women's preferences for men's facial masculinity are strongest under favorable ecological conditions. Scientific Reports, 9(1), 1-10.

Penke, L., \& Asendorpf, J. B. (2008). Beyond global sociosexual orientations: a more differentiated look at sociosexuality and its effects on courtship and romantic relationships. Journal of Personality and Social Psychology, 95, 1113-1135.

Penton-Voak, I. S., Perrett, D. I., Castles, D. L., Kobayashi, T., Burt, D. M., Murray, L. K., \& Minamisawa, R. (1999). Menstrual cycle alters face preference. Nature, 399(6738), 741-742.

Penton-Voak, I. S., Jacobson, A., \& Trivers, R. (2004). Populational differences in attractiveness judgements of male and female faces: Comparing British and Jamaican samples. Evolution and Human Behavior, 25(6), 355-370.

Perrett, D. I., Lee, K. J., Penton-Voak, I., Rowland, D., Yoshikawa, S., Burt, D. M., Henzi, S. P., Castles, D. L., \& Akamatsu, S. (1998).
Effects of sexual dimorphism on facial attractiveness. Nature, 394(6696), 884-887.

Rennels, J. L., Bronstad, P. M., \& Langlois, J. H. (2008). Are attractive men's faces masculine or feminine? The importance of type of facial stimuli. Journal of Experimental Psychology: Human Perception and Performance, 34(4), 884-893.

Rhodes, G., Chan, J., Zebrowitz, L. A., \& Simmons, L. W. (2003). Does sexual dimorphism in human faces signal health?. Proceedings of the Royal Society of London. Series B, 270(S1), S93-S95.

Saribay, S. A., Biten, A. F., Meral, E. O., Aldan, P., Třebický, V., \& Kleisner, K. (2018). The Bogazici face database: standardized photographs of Turkish faces with supporting materials. PLoS One, 13(2), e0192018. https://doi.org/10.1371/journal.pone.0192018.

Scott, I. M., Clark, A. P., Josephson, S. C., Boyette, A. H., Cuthill, I. C., Fried, R. L., ... \& Honey, P. L. (2014). Human preferences for sexually dimorphic faces may be evolutionarily novel. Proceedings of the National Academy of Sciences, 111(40), 14388-14393.

Thornhill, R., \& Gangestad, S. W. (1999). Facial attractiveness. Trends in Cognitive Sciences, 3(12), 452-460.

Thornhill, R., \& Gangestad, S. W. (2006). Facial sexual dimorphism, developmental stability, and susceptibility to disease in men and women. Evolution and Human Behavior, 27(2), 131-144.

Tiddeman, B., Burt, M., \& Perrett, D. (2001). Prototyping and transforming facial textures for perception research. IEEE Computer Graphics and Applications, 21, 42-50. https://doi.org/ 10.1109/38.946630.

Publisher's Note Springer Nature remains neutral with regard to jurisdictional claims in published maps and institutional affiliations. 